\title{
Propiedades sismo resistentes del tapial y la quincha: Arquitectura vernácula sostenible
}

Dennis Anthony Pérez Sánchez dperezsan@ucv.edu.pe Escuela de arquitectura Universidad Cesar Vallejo ORCID: 0000-0002-5761-9942

\author{
Jacqueline Bartra Gómez \\ jbartrag@ucv.edu.pe \\ Escuela de Arquitectura \\ Universidad Cesar Vallejo \\ ORCID: 0000-0002-2745-1587
}

\section{RESUMEN}

Esta investigación busca evaluar y describir las propiedades sismo resistentes del tapial y la quincha. Siendo el tipo de investigación básica, no experimental, el diseño del estudio se dio mediante revisiones fundadas en investigaciones científicas, que vienen a ser la herramienta principal para la síntesis de información, aumentando la validez y credibilidad de las conclusiones sacadas del estudio a los trabajos revisados, resolviendo áreas donde la investigación y análisis es primordial. En cuanto a las propiedades sismo resistentes del tapial y la quincha, donde según lo analizado, este depende mucho de las cualidades constructivas que se emplean, como lo son, el complementar a los materiales de estos sistemas constructivos, con estabilizadores naturales o comerciales, de igual manera escogiendo y evaluando un buen refuerzo sea interno o externo, para así, de esta manera se garantice la integridad tanto de la construcción como de quienes lo habitan. Por tal razón es primordial tomar en cuenta estos aspectos, evaluando también el impacto social, cultural, económico y ambiental que estos generan.

Palabras clave: Arquitectura, técnica de la construcción, refuerzo, seguridad. 


\title{
Earthquake resistant properties of the rammed earth and quincha: vernacular sustainable architecture.
}

\begin{abstract}
This research seeks to evaluate and describe the earthquake-resistant properties of the rammed earth and quincha. Being the type of basic research, not experimental, the design of the study was given through reviews based on scientific research, the analysis was carried out based on authentic primary studies, which become the main tool for the synthesis of information, increasing the validity and credibility of the conclusions drawn from the study to the reviewed works, solving areas where research and analysis are essential. Regarding the earthquake-resistant properties of the mud and the quincha, where according to what has been analyzed, this depends a lot on the constructive qualities that are used, such as complementing the materials of these constructive systems, with natural or commercial stabilizers, of, In the same way, choosing and evaluating a good internal or external reinforcement, to guarantee the integrity of both the construction and those who inhabit it. For this reason, it is essential to take these aspects into account, also evaluating the social, cultural, economic, and environmental impact that they generate.
\end{abstract}

Keywords: Architecture, construction technique, reinforcement, security.

Artículo recibido: 03 nov. 2020 Aceptado para publicación: 07 dic. 2020

Correspondencia dperezsan@ucv.edu.pe Conflictos de Interés: Ninguna que declarar 


\section{INTRODUCCIÓN}

Las construcciones de tierra se encuentran actualmente en todo el mundo, especialmente en los países con mayores necesidades habitacionales y menores recursos, como África, Oriente Medio y Latinoamérica. Es precisamente en América Latina, especialmente en Bolivia, donde se pueden encontrar ejemplos más grandes de edificaciones que utilizaban el suelo como principal material constructivo, esto ocurre especialmente en las zonas rurales, como lo muestra el censo de población y vivienda (2012), de 1992 a 2012, hubo una reducción porcentual del 27,6\%.

Cuadro 01. Porcentaje de construcciones con paredes de tierra en zona rural.

$\begin{array}{llll}\text { Año censal } & 1992 & 2001\end{array}$

Construcciones con paredes de tierra.

$65 \% \quad 52.8 \%$ $37,4 \%$

Fuente: elaboración propia

Esto se debe a diferentes factores, en los que se puede destacar, los factores sísmicos y la característica evolutiva de nuestra sociedad actual, que tiene preferencia por construcciones donde se usan como materiales principales el hormigón y los ladrillos, también llamado "material noble", que han ido reemplazando a los materiales tradicionales.

Por otro lado, muchos han asociado la arquitectura de tierra, con ideas de pobreza, atraso y peligro, sin tomar en cuenta las virtudes de este material, que solo necesita soporte técnico, basado en un adecuado sistema normativo, que permita su correcta implementación. La tierra se ha utilizado como material constructivo desde hace mucho tiempo, sobre todo por ser un material presente en todos lados y de fácil acceso, un claro ejemplo a nivel mundial viene a ser la ciudad de shibam en Yemen, considerado patrimonio de la Humanidad de la Unesco, que cuenta con edificios que llegan hasta los 14 pisos, construidos con tierra en distintas técnicas, los datos más antiguos en los que se da a conocer su existencia, datan del siglo II d.c. Lo increíble de las construcciones realizadas con este material, es su registro y duración a lo largo de todo este tiempo, lo que expone las grandes virtudes que tiene.

Las cualidades de sistemas constructivos como la quincha y tapial, como material sostenible son muchas, por lo que se espera que las investigaciones, el interés de la población y de los profesionales siga en aumento, para fomentar e impulsar este tipo de 
técnicas autóctonas de construcción, en el siglo XXI la conciencia ambiental está en aumento, el cual puede garantizar una buena proyección a futuro.

Como Formulación del Problema principal tenemos si el tapial y la quincha como sistemas constructivos sostenibles, son seguros ante fuerzas sísmicas este articulo tiene como objetivo Evaluar las propiedades sismo resistentes del tapial y la quincha como sistemas constructivos sostenibles.

\section{ESTRATEGIAS METODOLÓGICAS O MATERIALES Y MÉTODOS}

La naturaleza de la investigación será básica, no experimental; y se realizarán revisiones documentales, basadas en el análisis de estudios científicos. Por lo tanto, resultan ser herramientas útiles para la síntesis de la información científica disponible, validar las conclusiones e identificar aspectos en donde se necesite más investigación como complemento.

La recolección de datos se desarrolló gracias a la revisión bibliográfica de artículos, en donde el tema principal de investigación fueron las propiedades sismo resistentes del tapial o tierra apisonada y la quincha. Como punto de partida, se revisaron títulos y resúmenes de las referencias encontradas, lo que sirvió para la elección de las informaciones más resaltantes. Seguidamente, se procedió a la revisión de textos completos seleccionados para ratificar su elección. Para la revisión sistemática de los 10 artículos seleccionados se utilizó la técnica analítica, donde se evaluó cada uno de los estudios para extraer la información más relevante para realizar el respectivo análisis. 


\section{RESULTADOS Y DISCUSIÓN}

\section{RESULTADOS}

Estudios revisados sobre Análisis comparativo sismo resistente del tapial y la quincha.

\section{DATOS DE LA PUBLICACIÓN}

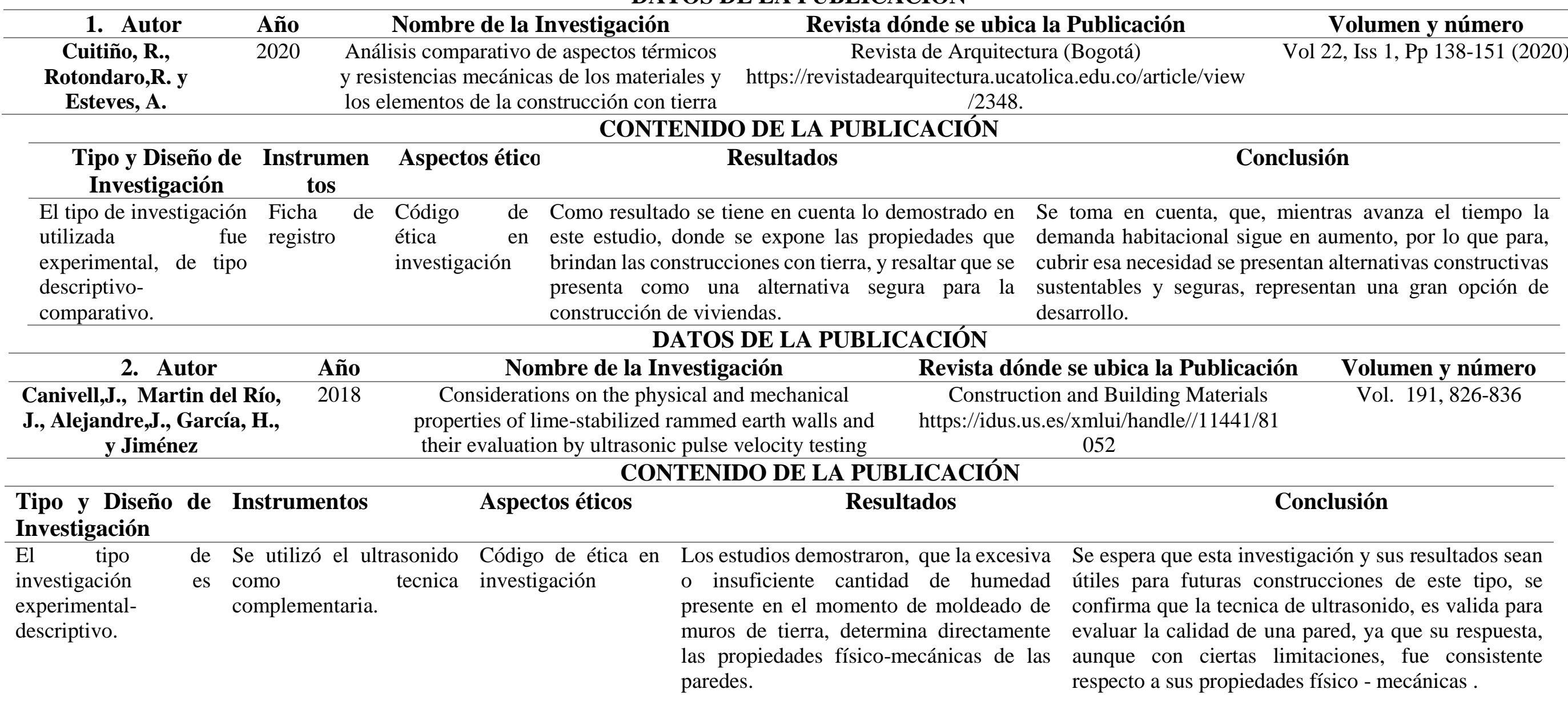




\section{DATOS DE LA PUBLICACIÓN}

\begin{tabular}{|c|c|c|c|c|c|}
\hline \\
\hline 3. Autor & Año & \multirow{2}{*}{\multicolumn{2}{|c|}{$\begin{array}{l}\text { Nombre de la Investigación } \\
\text { Experimental study on the } \\
\text { shaking table tests of a modern } \\
\text { inner-reinforced rammed earth } \\
\text { structure }\end{array}$}} & Revista dónde se ubica la Publicación & Volumen y número \\
\hline Zhou,T., Liu, B. & 2019 & & & Construction and Building Materials & $\begin{array}{l}\text { Apr2019, Vol. 203, p567-578. } \\
\text { 12p }\end{array}$ \\
\hline \multicolumn{6}{|c|}{ CONTENIDO DE LA PUBLICACIÓN } \\
\hline $\begin{array}{l}\text { Tipo y Diseño c } \\
\text { Investigación }\end{array}$ & Instrumentos & Aspectos éticos & & Resultados & Conclusión \\
\hline $\begin{array}{l}\text { El tipo de } \\
\text { investigación } \\
\text { es } \\
\text { experimental- } \\
\text { descriptivo. }\end{array}$ & $\begin{array}{l}\text { Se utilizó como } \\
\text { prueba la mesa } \\
\text { vibratoria. }\end{array}$ & $\begin{array}{l}\text { Código de ética en } \\
\text { investigación }\end{array}$ & $\begin{array}{l}\text { Los estudios } \\
\text { someter este } \\
\text { reforzando los } \\
\text { varillas de ba } \\
\text { resulta ser un b } \\
\text { esta manera se } \\
\text { cargas sismicas }\end{array}$ & $\begin{array}{ll}\text { mostraron, que al } & \text { Después de un } \\
\text { o de construcciones } & \text { construcción en u } \\
\text { ros interiormente, con } & \text { la mesa vibratoria } \\
\text { ú, madera o metal, } & \text { refuerzos interno } \\
\text { n refuerzo y aporta de } & \text { apisonada, po } \\
\text { idad y resistencia ante } & \text { ampliamente edi } \\
& \text { Sistema contructi }\end{array}$ & $\begin{array}{l}\text { revisión general del proceso de } \\
\text { sitio y los resultados de las pruebas de } \\
\text { s fácil sacar conclusiones de que estos } \\
\text { on adecuados para edificios de tierra } \\
\text { lo que se incentiva a construir } \\
\text { aciones que utlicen el tapial como } \\
\text { principal. }\end{array}$ \\
\hline
\end{tabular}

\section{DATOS DE LA PUBLICACIÓN}

\begin{tabular}{|c|c|c|c|c|c|}
\hline 4. Autor & & Año & Nombre de la Investigación & Revista dónde se ubica la Publicación & Volumen y número \\
\hline Cuitiño,G. & Esteves,A., & 2015 & Análisis de la transmitancia térmica & Centro Científico Tecnológico & 67 (537), art. no. e063 \\
\hline $\begin{array}{l}\text { Maldonado. } \\
\text { Rotondaro,R. }\end{array}$ & G. & & $\begin{array}{l}\text { y resistencia al impacto de los } \\
\text { muros de quincha }\end{array}$ & CONICET, en Mendoza-Argentina & \\
\hline
\end{tabular}

\begin{tabular}{|c|c|c|c|c|}
\hline \multicolumn{5}{|c|}{ CONTENIDO DE LA PUBLICACIÓN } \\
\hline Tipo de Investigación & Instrumentos & Aspectos éticos & Resultados & Conclusión \\
\hline $\begin{array}{c}\text { El tipo de } \\
\text { investigación }\end{array}$ & $\begin{array}{l}\text { Se utilizó el } \\
\text { hoque blando para }\end{array}$ & $\begin{array}{l}\text { Código de } \\
\text { ética en }\end{array}$ & $\begin{array}{l}\text { Los paneles de quincha fueron } \\
\text { construidos y probados a golpes }\end{array}$ & $\begin{array}{l}\text { La investigación demuestra que, } \\
\text { el comportamiento térmico y }\end{array}$ \\
\hline $\begin{array}{l}\text { es } \\
\text { experimental- } \\
\text { descriptivo. }\end{array}$ & nedir la resitencia & investigación & $\begin{array}{l}\text { suaves, en escala 1: 1. La } \\
\text { deformación permanente en todos } \\
\text { los casos fue inferior a los } 3,6 \mathrm{~mm} \\
\text { exigidos por la norma actual. Esto }\end{array}$ & $\begin{array}{l}\text { estructural, sumado a las } \\
\text { características de ser económico, } \\
\text { culturalmente representativo, y } \\
\text { con bajo impacto ambiental, }\end{array}$ \\
\hline
\end{tabular}


implica que no hubo convierten a la quincha como una deformaciones apreciables que buena opción de sistema sufrieron los cerramientos, dando constructivo para las viviendas.

seguridad a una vivienda.

\section{DATOS DE LA PUBLICACIÓN}

\begin{tabular}{|c|c|c|c|c|}
\hline 5. Autor & Año & Nombre de la Investigación & Revista dónde se ubica la Publicación & Volumen y número \\
\hline Cuitiño,G. Esteves,J., & 2020 & $\begin{array}{l}\text { El sistema constructivo de la } \\
\text { quincha en zonas rurales del Norte } \\
\text { de Mendoza (Argentina) }\end{array}$ & $\begin{array}{l}\text { Estoa. Revista de la Facultad } \\
\text { Arquitectura y } \\
\text { Universidad de Cuenca }\end{array}$ & Estoa vol.9 no.17, p93 \\
\hline
\end{tabular}

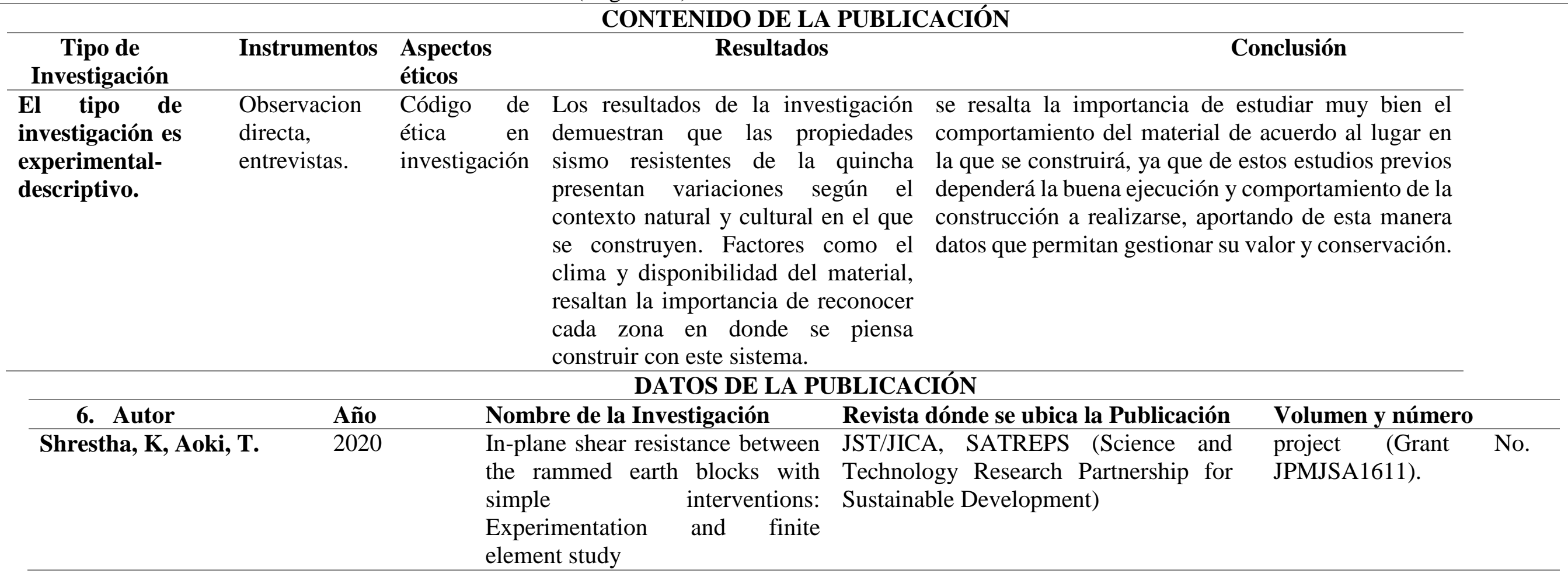


CONTENIDO DE LA PUBLICACIÓN

Tipo de Investigación

\section{El tipo de} investigación es experimental
Instrumentos Aspectos éticos

ensayos de Código

resistencia a la ética rotura por tracción investigación

y compresión en

muestras extraídas

de núcleos

cilíndricos

(probetas)
Resultados

de Se discute la efectividad de una

en intervención simple en los muros, con la inserción de un anclaje de hormigón armado en la interfaz del bloque de tierra apisonada, como medida de refuerzo. Los resultados mostraron que la resistencia al corte de la muestra reforzada aumentó en un $12,3 \%$

\section{Conclusión}

Para el análisis de este trabajo de investigación, se tomó en cuenta tanto las observaciones experimentales como los cálculos numéricos, que mostraron la eficacia de la técnica de intervención propuesta para mejorar la resistencia al corte y retrasar de esta manera el deslizamiento a lo largo de la interfaz de las juntas en los muros de tapial.

\section{DATOS DE LA PUBLICACIÓN}

\begin{tabular}{|c|c|c|c|c|}
\hline 7. Autor & Año & Nombre de la Investigación & Revista dónde se ubica la & Volumen y número \\
\hline Yang, X. , Wang,H. & 2018 & $\begin{array}{l}\text { Seismic Behavior of Rammed Earth } \\
\text { Walls with Precast Concrete Tie } \\
\text { Columns }\end{array}$ & $\begin{array}{l}\text { Advances in Material } \\
\text { Engineering. }\end{array}$ & 3/13/2018, p1-10. 10p. \\
\hline
\end{tabular}

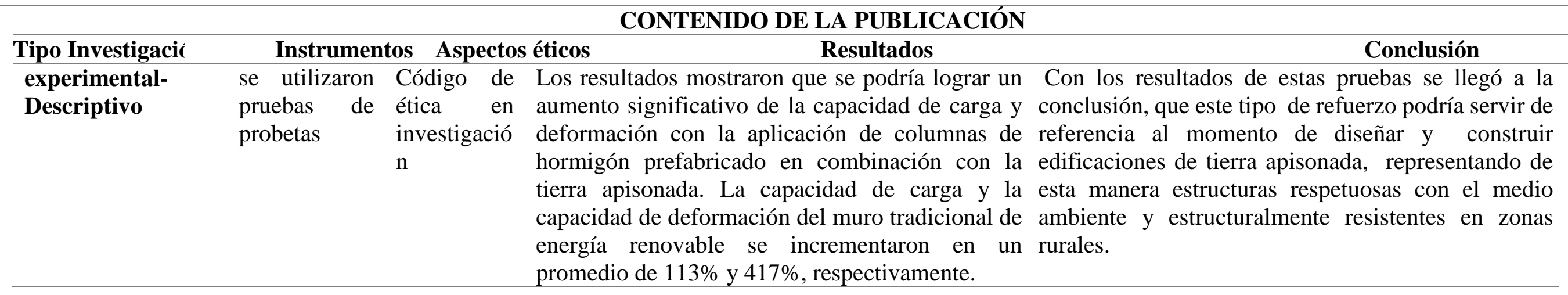


DATOS DE LA PUBLICACIÓN

Revista dónde se ubica la Publicación

Advances
Engineering.

in

\begin{tabular}{cc} 
8. Autor & Año \\
\hline Zhang, K., Lu, B., Wang, Y., & 2019
\end{tabular}

Lei, Z., Yang, Z
Experimental Strength of Earth-Based

Construction Materials in Different

Regions of China
Nombre de la Investigación

\section{u}

\section{ica la $\mathbf{P}$}

\section{Resultados}

\section{éticos}

Investigación

El tipo de

investigación es experimental-

Descriptivo

$\begin{array}{llr}\text { se } & \text { Código } & \text { de } \\ \text { utilizaron } & \text { ética } & \text { en } \\ \text { pruebas de } & \text { investigación } \\ \text { probetas } & & \end{array}$

Los resultados expusieron, que, de las muestras tomadas en diferentes

regiones de china, la tierra negra y la tierra amarilla del noroeste de la región, parecen ser más favorables como material de construcción, ya que aportan propiedades beneficiosas al momento del uso constructivo que se le dé, por lo que estos resultados pueden servir como referencia para la selección de material en futuras construcciones.

\section{DATOS DE LA PUBLICACIÓN}

$\begin{array}{cc}\text { 9. Autor } & \text { Año } \\ \text { Khadka, B } & 2020\end{array}$

Khadka, B

\section{Nombre de la Investigación}

Rammed earth, as a sustainable and structurally safe green building: A housing solution in the era of global warming and climate change

\section{Revista dónde se ubica la Publicación}

\section{Asian Journal of Civil Engineering}

A

\section{CONTENIDO DE LA PUBLICACIÓN}

\section{Tipo Investigación Instrumentos Aspectos éticos}

Resultados
Volumen y número

Science \& 3/3/2019, p1-9.9p. significativas en la composición de los materiales de construcción a base tierra, y que éstas mismas influyen directamente el comportamiento sismo resistente de la construcción.

\begin{tabular}{|c|c|c|c|c|}
\hline $\begin{array}{l}\text { El tipo de } \\
\text { investigación es } \\
\text { experimental- } \\
\text { descriptivo }\end{array}$ & $\begin{array}{l}\text { Prototipos de } \\
\text { vivienda }\end{array}$ & $\begin{array}{ll}\text { Código de } \\
\text { ética en } \\
\text { investigación }\end{array}$ & $\begin{array}{l}\text { Los resultados mostraron que, las muestras estabilizadas con } \\
\text { cemento (cemento al 5\%) tuvieron mejores resultados } \\
\text { generales que las muestras estabilizadas con estiércol y sin } \\
\text { estabilizar. Además, la comparación de costos mostró que la } \\
\text { pared de tierra apisonada de } 360 \mathrm{~mm} \text { de espesor era un 10- } \\
15 \% \text { más barata que la pared de ladrillo de } 230 \mathrm{~mm} \text { de } \\
\text { espesor }\end{array}$ & $\begin{array}{l}\text { La intención principal del estudio es } \\
\text { proporcionar una solución en forma } \\
\text { sostenible, exponiendo la capacidad de } \\
\text { refuerzo sísmico que presentan las } \\
\text { construcciones con materiales alternativos } \\
\text { como la tierra apisonada donde se utiliza } \\
\text { subsuelo natural como constituyente } \\
\text { primario. }\end{array}$ \\
\hline
\end{tabular}


DATOS DE LA PUBLICACIÓN

\begin{tabular}{|c|c|c|c|c|c|c|}
\hline \\
\hline 10. Autor & Año & \multirow{2}{*}{\multicolumn{2}{|c|}{$\begin{array}{l}\text { Nombre de la Investigación } \\
\text { Seismic behaviour of rammed earth } \\
\text { walls: A time history analysis }\end{array}$}} & \multicolumn{2}{|c|}{$\begin{array}{l}\text { Revista dónde se ubica la Publicación } \\
\text { Lecture Notes in Civil Engineering }\end{array}$} & \multirow{2}{*}{$\begin{array}{l}\text { Volumen y número } \\
\text { Volume 54, 2020, Pages } \\
\text { 143-148. }\end{array}$} \\
\hline Bui, Q., \& Bui, T. & 2020 & & & Lecture Notes in Civil En & gineering & \\
\hline \multicolumn{7}{|c|}{$\begin{array}{l}\text { CONTENIDO DE LA PUBLICACIÓN } \\
\end{array}$} \\
\hline $\begin{array}{l}\text { Tipo } \\
\text { Investigación }\end{array}$ & Instrumentos & $\begin{array}{l}\text { Aspectos } \\
\text { éticos }\end{array}$ & Resul & $\operatorname{tados}$ & & nclusión \\
\hline $\begin{array}{l}\text { El tipo de } \\
\text { investigación es } \\
\text { experimental- } \\
\text { descriptivo }\end{array}$ & $\begin{array}{l}\text { Análisis de } \\
\text { Probetas. }\end{array}$ & $\begin{array}{l}\text { Código de } \\
\text { ética en } \\
\text { investigación }\end{array}$ & $\begin{array}{l}\text { El modelo se escaló } \\
\text { para evaluar los daî } \\
\text { intensidades de car } \\
\text { registrando los dañ } \\
\text { discutió el compor } \\
\text { fue favorable y nos } \\
\text { este tipo de }\end{array}$ & $\begin{array}{l}\text { en diferentes amplitudes } \\
\text { ños después de diferentes } \\
\text { gas sismicas.se concluyó } \\
\text { os que se pudo lograr y se } \\
\text { tamiento sísmico, el cual } \\
\text { da una visión segura de } \\
\text { construcciones. }\end{array}$ & $\begin{array}{r}\text { Con los resu } \\
\text { concluye } \\
\text { resultan s } \\
\text { sostenible } \\
\text { construccion } \\
\text { cuidado y pr } \\
\text { en c }\end{array}$ & $\begin{array}{l}\text { s obtenidos anteriormente se } \\
\text { las edificaciones con tierra } \\
\text { na alternativa vernácula - } \\
\text { tima y segura para futuras } \\
\text { acentivando de esta manera el } \\
\text { vación cultural de los lugares } \\
\text { e se implementarán. }\end{array}$ \\
\hline
\end{tabular}





\section{DISCUSION}

De acuerdo a la revisión de los artículos, encontramos que del 100\% de artículos estudiados, el 30\% de artículos presentados son de América latina, como lo son Argentina y Colombia, el $70 \%$ restante engloba estudios realizados en Asia y Norte América. En lo que respecta a los diseños y tipos de estudios el 100\% son descriptivos.

En lo que concierne a las propiedades sismo resistentes del tapial y la quincha se puede resaltar que Cuitiño,G. Esteves,J., exponen que las propiedades sismo resistentes de la quincha presentan variaciones según el contexto natural y cultural en el que se construyen, por lo que es importante reconocer cada zona, estudiar factores como el clima y disponibilidad del material, para tomar decisiones tanto de diseño, como de construcción.. Por su parte Zhou,T., Liu, B., demuestran la importancia que representa reforzar interiormente los muros de tierra apisonada con varillas de madera, bambú o de metal, para asegurar una construcción firme y segura ante posibles cargas sísmicas en el futuro.

De igual forma Cuitiño,G. Esteves,A., Maldonado. G. y Rotondaro,R., nos mencionan en su investigación que las construcciones realizadas con paredes de quincha resisten de manera favorable a cargas sísmicas, sin sufrir daños considerables, por lo que se expone a este sistema constructivo como una alternativa segura. Además, Shrestha, K, Aoki, T. nos muestran la efectividad de una intervención simple en los muros, con la inserción de un anclaje de hormigón armado en los muros de tierra apisonada, como medida de refuerzo demostrando así la resistencia al corte frente a cargas sísmicas.

\section{CONCLUSIÓN O CONSIDERACIONES FINALES}

Existen numerosos estudios que demuestran los beneficios que presentan las construcciones de tapial y quincha ante fuerzas sísmicas, factores como; la diferencia de ejecución, elección de materiales y técnicas constructivas adecuadas, son los principales componentes que aportan dichas propiedades. Es preciso señalar también, que, la ubicación geográfica o emplazamiento de la construcción, resulta ser uno de los puntos más importantes a tomar en cuenta, ya que se tiene que evaluar meticulosamente condicionantes, como el clima, disponibilidad de materiales e influencias socio-culturales propias de cada lugar.

Actualmente la preocupación por el cuidado del medio ambiente y reducir la huella de carbono en el ámbito constructivo, es muy grande, por lo que representan una opción constructiva sustentable, por el bajo impacto ambiental, y económico que este genera. Es 
por ello que el interés y estudio de estos sistemas constructivos resulta ser de gran importancia, por los beneficios intrínsecos que estos representan en distintos ámbitos.

\section{LISTA DE REFERENCIAS}

María Guadalupe Cuitiño-Rosales, Rodolfo Rotondaro, \& Alfredo Esteves. (2020). Análisis comparativo de aspectos térmicos y resistencias mecánicas de los materiales y los elementos de la construcción con tierra. Revista de Arquitectura (Bogotá), 22(1), 138-151. https://doi.org/10.14718/RevArq.2020.2348

Canivell, J., Martín del Río, J. J., Alejandre Sánchez, F. J., García Heras, J., \& Jiménez Aguilar, A. (2018). Considerations on the physical and mechanical properties of lime-stabilized rammed earth walls and their evaluation by ultrasonic pulse velocity testing.

Zhou, T., \& Liu, B. (2019). Experimental study on the shaking table tests of a modern inner-reinforced rammed earth structure. Construction \& Building Materials,203,567-578. https://doi.org/10.1016/j.conbuildmat.2019.01.070

Cuitiño, G., Esteves, A., Maldonado, G., \& Rotondaro, R. (2015). Analysis of thermal transmittance and resistance to soft shock in wattle walls. [Análisis de la transmitancia térmica y resistencia al impacto de los muros de quincha] Informes De La Construccion, 67(537) doi:10.3989/ic.12.082

Esteves, Matías Jose, \& Cuitiño, Guadalupe. (2020). El sistema constructivo de la quincha en zonas rurales del Norte de Mendoza (Argentina). Estoa. Revista de la Facultad de Arquitectura y Urbanismo de la Universidad de Cuenca,9(17), 153169. https://doi.org/10.18537/est.v009.n017.a08

Yang, X., \& Wang, H. (2018). Seismic Behavior of Rammed Earth Walls with Precast Concrete Tie Columns. Advances in Materials Science \& Engineering, 1-10. https://doi.org/10.1155/2018/9739853

Zhang, K., Lu, B., Wang, Y., Lei, Z., \& Yang, Z. (2019). Experimental Strength of EarthBased Construction Materials in Different Regions of China. Advances in Materials Science \& Engineering, 1-9. https://doi.org/10.1155/2019/8130743

Khadka, B. (2020). Rammed earth, as a sustainable and structurally safe green building: A housing solution in the era of global warming and climate change. Asian Journal of Civil Engineering, 21(1), 119-136. doi:10.1007/s42107-019-00202-5 Retrieved from www.scopus.com 
Bui, Q. -., \& Bui, T. -. (2020). Seismic behaviour of rammed earth walls: A time history analysis doi:10.1007/978-981-15-0802-8_19 Retrieved from www.scopus.com

Shrestha, K. C., Aoki, T., Miyamoto, M., Wangmo, P., \& Pema. (2020). In-plane shear resistance between the rammed earth blocks with simple interventions: Experimentation and finite element study. Buildings, 10(3) doi:10.3390/buildings 10030 